\title{
Références bibliographiques du dossier : « Le métier d'enseignant en Europe »
}

\section{Marie-France Pamart}

\section{Q OpenEdition}

1 Journals

Édition électronique

URL : https://journals.openedition.org/ries/1929

DOI : 10.4000/ries. 1929

ISSN : 2261-4265

Éditeur

France Education international

\section{Édition imprimée}

Date de publication : 1 septembre 2002

Pagination : 109-122

ISBN : 978-285420-554-1

ISSN : 1254-4590

Référence électronique

Marie-France Pamart, « Références bibliographiques du dossier : " Le métier d'enseignant en Europe » », Revue internationale d'éducation de Sèvres [En ligne], 30 | septembre 2002, mis en ligne le 24 novembre 2011, consulté le 06 juillet 2021. URL : http://journals.openedition.org/ries/1929 ; DOI https://doi.org/10.4000/ries.1929 


\title{
Références bibliographiques
}

\section{Marie-France Pamart}

La bibliographie proposée présente un choix de textes récents, postérieurs à 1996, référencés dans la base de données du centre de ressources du CIEP ou consultables sur l'Internet. Elle est organisée autour de quatre axes qui correspondent aux diverses facettes du thème de ce dossier sur les enseignants : l'état des lieux, la carrière, le métier, les perspectives.

\section{État des lieux ou radioscopie des enseignants}

\begin{abstract}
BOURDONCLE Raymond éd., DEMAILLY Lise éd, Les professions de l'éducation et de la formation, Villeneuve d'Ascq, Presses universitaires du Septentrion (Les métiers de la formation), 1998, $480 \mathrm{p}$.

Aujourd'hui, les différentes institutions de transmission de savoirs et les activités d'éducation et de formation connaissent une forte déstabilisation qui affecte à la fois les organisations et les pratiques professionnelles. Ainsi, les métiers de l'éducation et de la formation se trouvent pris entre une exigence de rationalisation, une volonté de professionnalisation, un consensus sur leur caractère socialement prioritaire et des incertitudes conflictuelles sur leurs objectifs d'action. Toutes ces questions sont débattues dans cet important ouvrage auquel un grand nombre de professionnels de l'éducation ont apporté leur contribution.
\end{abstract}

COMMUNAUTÉ EUROPÉENNE : Commission; EUROSTAT (Office statistique); EURYDICE (Réseau européen d'information sur l'éducation), Les chiffres clés de l'éducation en Europe: 99, Office des publications officielles des Communautés européennes/Luxembourg, 2000, 260 p.

Cette quatrième édition couvre maintenant 29 pays européens (UE et les pays en pré-adhésion) et s'enrichit de nombreux nouveaux indicateurs. Un chapitre entier est ainsi consacré aux technologies de l'information et de la communication dans les systèmes éducatifs; un autre décrit la formation des enseignants des niveaux primaire et secondaire en notant que la formation de niveau universitaire se généralise dans beaucoup de pays européens; un autre rassemble désormais toutes les informations relatives à l'apprentisage des langues étrangères auparavant réparties sur plusieurs chapitres. Globalement, ceux-ci restent structurés par niveau d'enseignement mais ne comportent pas d'indicateurs ni de descriptions spécifiques sur les systèmes de formation professionnelle initiale et continue (cf. Chiffres clés sur la formation professionnelle dans l'UE); de même, on ne trouve aucune donnée relative au financement de l'éducation ( $c f$. la série : Questions clés...).

DIETSCH Bruno, MALEGUE Claude, MIGEON Martine, «Connaissance des enseignants : photographie du corps enseignant à la fin des années quatre-vingtdix ", Éducation et formations, $n^{\circ} 56$, juin 2000, p. 13-85.

Dans la continuité des numéros précédents, celui-ci présente une radiographie du corps enseignant (premier et second degrés) à l'aube de l'an 2000. Le numéro rend ainsi compte de quelques-unes de ses caractéristiques majeures, à travers un panorama d'ensemble qui s'intéresse notamment aux conditions de travail et à la maîtrise du métier. 
MARESCA Bruno, CREDOC (Centre de recherche pour l'étude et l'observation des conditions de vie), "Le métier d'enseignant: contributions à l'analyse de l'univers professionnel des maîtres et des professeurs ", Cahiers de recherche, $n^{\circ} 140$, novembre 1999, 84 p., tabl., bibliogr.

Suite de contributions sur le métier des enseignants dans les écoles maternelles et primaires, les collèges et les lycées de l'enseignement public : le contexte socio-économique de l'exercice de la profession enseignante, les attentes des parents vis-à-vis de l'école, les caractéristiques sociales du corps enseignant, l'organisation du travail des enseignants, les satisfactions, difficultés, représentations du métier, la fonction managériale des chefs d'établissements du secondaire.

Rapport mondial sur l'éducation 1998 : les enseignants et l'enseignement dans un monde en mutation, Paris, UNESCO, 1998, 174 p., tabl., graph.

Cette $4^{\mathrm{e}}$ édition porte sur la profession enseignante qui compte 57 millions de membres dans le monde. Elle recense les tendances et évolutions récentes de l'éducation et des politiques éducatives qui ont des incidences sur les enseignants, en particulier sur leur statut, leurs conditions de travail, leur niveau de formation et leurs possibilités de perfectionnement. En annexe se trouvent les indicateurs de l'éducation dans le monde avec des tableaux récapitulatifs par pays, ainsi qu'une liste des rapports, publications et périodiques de l'UNESCO concernant l'éducation pendant la période 1995-1997.

VANISCOTTE Francine, "L'enseignant des écoles de l'Europe», Perspectives documentaires en éducation, $n^{\circ}$ 46/47, octobre 1999, p. 115-124.

Petite typologie des systèmes éducatifs aujourd'hui en Europe accompagnée de tableaux comparatifs sur la formation initiale des enseignants du premier degré dans les quinze pays de l'UE.

\section{La carrière des enseignants}

\section{Formation initiale et continue : les évolutions récentes}

BOTTERY Mike, WRIGHT Nigel, Teachers and the State: towards a directed profession, Londres, Routledge (Routledge research in education), 2000, 175 p., index, bibliogr.

Dans le contexte actuel du débat sur la modernisation de la profession d'enseignant, cet ouvrage examine le statut, la formation initiale et continue des enseignants du Royaume-Uni.

BOUVIER Alain coord., OBIN Jean-Pierre coord., La formation des enseignants sur le terrain, Paris, Hachette Éducation, (Former, organiser pour enseigner), 1998, $253 p$.

L'ensemble des contributions de cet ouvrage vise à établir une sorte de dialectique des rapports entre savoirs et pratiques. Quelle forme doit prendre l'alternance entre le terrain et le centre de formation pour faciliter la construction des compétences professionnelles? Comment amener le formé à prendre conscience de ses manières de faire et faciliter le regard de chacun sur ses propres démarches? Et un nouveau profil de formateur apparait : du formateur ingénieur-didacticien au formateur médiateur-facilitateur de l'analyse par les formés de leur vécu de formation. 
CANARIO Rui, CORREIA José Alberto, «Enseignants au Portugal : formation continue et enjeux identitaires", Éducation et sociétés, $n^{\circ}$ 4, février 1999, p. 131-142, bibliogr.

Au Portugal l'offre de formation continue pour les enseignants connait une croissance exponentielle depuis 1993. Inscrite simultanément dans une logique de contrôle par l'État et dans une logique de marché, elle a cessé d'être envisagée comme un droit pour devenir un devoir, souvent pénible. À partir de recherches récentes, les auteurs de l'article tentent d'analyser comment cette offre de formation s'articule avec les façons de concevoir l'activité professionnelle des enseignants, leur mode de socialisation professionnelle et les dynamiques de production identitaire.

CROS Françoise réd., "Innovation et formation des enseignants », Recherche et formation, $n^{\circ} 31$, décembre 1999, p. 5-112.

Ce premier numéro sur l'innovation porte sur la formation initiale et la formation continue des enseignants articulées au sein d'une même institution : les IUFM. Trois questions y sont plus précisément traitées : le comportement des enseignants face à l'innovation, les liens (antinomiques?) entre la formation des enseignants et l'innovation, les effets formateurs chez tout enseignant engagé dans un processus d'innovation.

\section{L'école à la page : formation continue et perfectionnement professionnel des enseignants, Paris, OCDE, 1998, 194 p., bibliogr.}

La formation continue et le perfectionnement professionnel des enseignants font désormais partie intégrante d'une évolution des systèmes éducatifs fondée sur l'élaboration de stratégies nouvelles et dynamiques au niveau de l'école. Les pays étudiés dans ce rapport de l'OCDE (Allemagne, États-Unis, Irlande, Japon, Luxembourg, Royaume-Uni, Suède) ont des traditions et des systèmes différents en matière d'éducation. Cependant, ils arrivent tous à la même conclusion : les méthodes de perfectionnement professionnel les plus efficaces combinent des activités sur place et hors de l'école. L'expérience de ces pays fait également ressortir la nécessité d'une identification plus systématique des besoins, d'une meilleure évaluation des programmes et d'une diffusion améliorée de ces évaluations.

Enseigner au collégial : une pratique professionnelle en renouvellement, SainteFoy, Canada, Conseil supérieur de l'éducation, 1997, 106 p., bibliogr.

Après l'étude des paramètres de la pratique professionnelle enseignante sont privilégiées quatre voies de développement : le renouvellement des pratiques départementales, la contribution de l'évaluation au développement professionnel, la reconnaissance des principaux enjeux liés à la qualification, enfin la reconnaissance de la diversité des pratiques.

FOSTER Rob, «Becoming a secundary teacher in France: a trainee perspective on recent developments in initial teacher training", Educational studies, vol. $26, n^{\circ} 1$, mars 2000 , p. 5-17.

Point de vue d'un formateur britannique sur la réforme de la formation des enseignants du secondaire en France. Cet analyse du rôle des IUFM conduit l'auteur à quelques comparaisons intéressantes avec d'autres pays d'Europe.

GAUTHIER Pierre-Louis coord., "La formation des enseignants : I. Des approches contrastées", Revue internationale d'éducation Sèvres, $n^{\circ}$ 20, décembre 1998, p. 33-151, bibliogr.

Ce premier numéro consacré à la formation des enseignants rassemble des contributions venues de quatre continents : Europe, Asie, Amérique du Nord et Afrique. Cette dimension internationale de l'analyse révèle la diversité des points de vue qui relève souvent de concep- 
tions différentes liées à l'histoire, aux traditions culturelles, au statut de l'école et de l'enseignant dans la société. Les entrées choisies par les divers auteurs et leurs approches personnelles du dossier permettent de replacer chaque système de formation dans son environnement. On voit cependant émerger de ces approches contrastées des thèmes communs à tous les systèmes de formation (recrutement, contenus des études, théorie et pratique, professionnalisation, etc.).

GAUTHIER Pierre-Louis coord., «La formation des enseignants : II. Des problématiques convergentes", Revue internationale d'éducation Sèvres, $n^{\circ} 21$, mars 1999, p. 19-143, bibliogr.

Ce deuxième numéro consacré à la formation des enseignants tente de dégager des problématiques communes à différents pays : le système de formation face aux bouleversements du corps social, la finalité de la formation, les nouveaux profils d'enseignants, les contenus de la formation et la professionnalisation. S'il y a convergence sur ces problèmes, les solutions restent spécifiques.

JACQUET-FRANCILLON François, CAREIL Yves, PEYRONIE Henri, RAYOU Patrick, AUDIGIER François, «Être et devenir professeur des écoles», Perspectives documentaires en éducation, $n^{\circ}$ 46/47, octobre 1999, p. 7-124.

Après un éclairage historique, ce dossier thématique apporte, avec les contributions de sociologues, psychologues et pédagogues, un regard neuf sur les enjeux de «l'école du XXI" siècle» et la formation des enseignants en IUFM.

"La formacion permanente del profesorado", Revista de educacion, $n^{\circ} 317$, décembre 1998, p. 7-142.

À partir des conclusions de la Conférence internationale sur l'éducation (UNESCO, Genève, 5 octobre 1996), ce dossier poursuit la réflexion sur la formation permanente des enseignants, particulièrement les enseignants du secondaire; quelques articles l'étendent à l'Europe.

NAGY Maria, ZULKUF ALTAN Mustafa, DAY Christopher W., TERHART Ewald, SIMPSON Mary, HAYWARD Louise, PELAGE Agnès, EVETTS Julia, "Teacher education", European journal of education, vol. 33, n 4, décembre 1998, p. 389-473.

La formation des enseignants dans les pays industrialisés est le thème central de cette livraison. Le point de départ de cette étude se trouve dans les résultats du projet pilote SIGMA de la Commission européenne qui furent présentés à la Conférence d'Osnabrück en juin 1995. Dans un des rapports, il ressort qu'il ne suffit pas de renforcer la formation telle qu'en ellemême, mais d'en redéfinir les besoins et les finalités et d'apporter de nombreuses innovations; certaines d'entre elles font l'objet des articles de ce numéro.

PERIER Pierre, "Devenir professeur des écoles : enquête auprès des débutants et anciens instituteurs ", Les Dossiers, $n^{\circ}$ 123, juin 2001, 167 p.

Issu d'une enquête menée en juin 2000 auprès d'un échantillon de 858 professeurs des écoles (pour moitié jeunes enseignants sortis des IUFM, pour moitié anciens instituteurs), ce dossier offre une radioscopie détaillée du corps des enseignants du premier degré. L'analyse porte sur l'accès à la fonction, l'exercice du métier, la formation en IUFM et la formation continue, les représentations de la fonction, du statut et du système éducatif. La dernière partie dresse une typologie des professeurs des écoles en fonction de leurs positions plus ou moins critiques sur leur statut, le métier, les élèves et l'institution.

«Principes et modalités d'organisation de la deuxième année de formation des enseignants et des conseillers principaux d'éducation stagiaires", Bulletin officiel de l'Education nationale, $n^{\circ} 15$, avril 2002, 18 p. téléchargées du site: www.education.gouv. fr/bo/2002/15/encart.htm 
Conforme au plan de rénovation de la formation des enseignants du 17 février 2001 et s'appuyant notamment sur le travail de Ph. Meirieu ("Cahier des charges de la deuxième année en IUFM", novembre 2001), ce texte organise la deuxième année dans les IUFM; il propose également le cahier des charges de cette deuxième année qui souligne les éléments du tryptique de formation (stages, enseignements, travail personnel et mémoire). En annexe, éléments d'aide à la réalisation du cahier des charges.

\section{ROBERT André D., TERRAL Hervé, Les IUFM et la formation des enseignants} aujourd'hui, Paris, PUF (Education et formation: formation permanente, éducation des adultes), 2000, $161 \mathrm{p}$.

Après dix années d'existence, il était bon de dresser un état des lieux des IUFM. Deux chercheurs en éducation se sont attelés à la tâche et ont produit cet ouvrage qui est le permier bilan historique, sociologique et pédagogique des IUFM. Dans une première partie, les auteurs rappellent les nombreux débats qui précédèrent la mise en place de cette institution, puis tout en poursuivant l'analyse du processus constitutif des IUFM, ils abordent leur mise en œuvre effective eu égard aux nombreuses contraintes qui se sont présentées aux réformateurs. Après cette mise en place, on assiste dans la troisième partie au déferlement de critiques venant à la fois de l'opinion publique et du milieu professionnel. La dernière partie traite du «devenir et de l'avenir» des IUFM, en insistant sur la question du professionnalisme.

TARDIF Maurice dir., LESSARD Claude dir., GAUTHIER Clermont dir., ALTET Marguerite, PERRENOUD Philippe, CALDERHEAD James, PINAR William F., THERRIEN Jacques, Formation des maîtres et contextes sociaux : perspectives internationales, Paris, PUF, (Éducation et formation: biennales de l'éducation), 1998, 290 p., bibliogr.

Cet ouvrage s'efforce de décrire, d'analyser et de comparer les réformes de la formation des enseignants entreprises depuis à peu près une décennie dans divers pays, sous l'angle de la professionnalisation de l'enseignement et de ses enjeux. Il veut également offrir une vision contrastée et critique de ce qui se passe dans chacun de ces pays en adoptant une perspective internationale ou transnationale et en mettant en évidence aussi bien les dimensions sociopolitiques des réformes que les différentes logiques à l'œuvre. Certaines contributions sont en anglais.

\section{Évaluation et gestion des enseignants}

ANSALLEM Jean-Claude, CADIS Pierre, "La déconcentration», Cahiers de l'Éducation, $n^{\circ}$ 6, octobre 1999, p. 2-13.

Ce dossier offre une vue générale sur la déconcentration de l'administration française et de l'Éducation nationale en particulier, avec un article plus précis sur la déconcentration du mouvement des personnels enseignants, récemment mise en place, vue à travers les impressions d'un proviseur.

DEMAILLY Lise réd., DASTE Pierre réd., «La gestion des compétences dans l'Éducation nationale», Recherche et formation, $n^{\circ} 30$, octobre 1999, p. 5-163, bibliogr.

Ce numéro est consacré à la gestion des compétences dans l'Éducation nationale en se centrant sur le groupe des enseignants de l'élémentaire et du secondaire et en effleurant celle des chefs d'établissement. Par contre, la gestion des inspecteurs et des personnels de l'admi- 
nistration n'est absolument pas traitée. L'ensemble des contributions proposées tourne autour du contenu de cette expression à la mode «la gestion des compétences» et les auteurs, universitaires, hauts fonctionnaires ou syndicalistes apportent des réponses diversifiées et divergentes à cette question. Quelques études de cas complètent le numéro.

GAYNOR Cathy, WORLD BANK, Decentralization of education: teacher management, Washington, World Bank, 1998, 76 p.

Réformant leur système éducatif, bon nombre de pays décentralisent l'administration et la gestion du personnel enseignant. Ce petit essai a l'ambition d'aider les politiques en ce domaine.

\title{
MONTEIL Jean-Marc, Propositions pour une nouvelle approche de l'évaluation
} des enseignants, juin 1999, 28 p. téléchargées du site www.education.gouv.fr

Ce rapport répond à une demande du ministre de l'Éducation nationale qui désire conduire une véritable politique de valorisation des ressources humaines à propos de la carrière des enseignants, ce qui induit, au préalable, une mission de réflexion, d'analyse et de propositions centrée en particulier sur les problèmes de notation.

\section{Le métier d'enseignant} Professionnalisation et pratiques réflexives

\begin{abstract}
ABDALLAH-PRETCEILLE Martine dir., PORCHER Louis dir., "Éthique, communication et éducation", Le Français dans le monde : recherches et applications, $n^{\circ}$ spécial, juillet 1999, 135 p.

L'enseignant se trouvant au carrefour d'une éthique de l'action et d'une éthique de la relation, la déontologie occupe une place importante dans l'exercice de son métier. Ce numéro s'attache ainsi aux enjeux et interrogations liés à l'éthique, à la relation entre éthique et langage et à celle qui caractérise l'éthique, la culture et l'éducation.
\end{abstract}

ALTET Marguerite réd., BOURDONCLE Raymond réd., "Formes et dispositifs de la professionnalisation", Recherche et formation, $n^{\circ} 35$, décembre 2000, p. 5-195.

Les travaux de recherche sur le processus de professionnalisation montrent qu'il prend des formes diverses et se met en place selon des dispositifs variés. "La professionnalisation des personnes, c'est-à-dire leur acquisition de connaissances et d'identité propres à la profession, a recours aussi bien à la socialisation qu'à la formation professionnelle»: cette phrase de l'éditorial montre bien les deux axes autour desquels vont s'orienter les articles de ce dossier, en privilégiant cependant la formation.

BARLOW Michel, Le métier d'enseignant : essai de définition, Paris, Anthropos (Education), 1999, 162 p., bibliogr., index.

Pour définir le métier d'enseignant, l'auteur le suit dans les étapes de sa stratégie: définition d'un projet, choix des objectifs, création de méthodes et de moyens pédagogiques, régulation de l'action par des valeurs de référence, évaluation en cours de route et à son terme. Ce parcours s'effectue à travers la confrontation avec d'autres métiers : artisan ou artiste, médecin ou pharmacien, magistrat ou homme politique. 
BORKO Hilda coord., PUTNAM Ralph coord., "Professional development and reform-based teaching", Teaching and teacher education, vol. 14, $n^{\circ} 1$, janvier 1998, p. 1-140.

Le thème de ce numéro porte sur la professionnalisation du métier d'enseignant au RoyaumeUni. Les diverses contributions analysent des aspects variés de ce changement comme la participation de l'Université, les réflexions des enseignants sur leurs propres pratiques, le rôle des enseignants expérimentés dans l'aide apportée aux plus novices.

BRAUN Jean-Paul éd., CASTINCAUD Florence éd., "Notre métier, notre identité », Cahiers pédagogiques, $n^{\circ} 380$, janvier 2000, p. 7-56.

Construction de l'identité professionnelle des enseignants, multiplicité des identités en relation avec la multiplicité des tâches et des pratiques pédagogiques, relations de parcours professionnels, récits de vie et biographie professionnelle : tels sont les thèmes développés dans ce numéro.

\section{CHARLES Frédéric, CLEMENT Jean-Paul, Comment devient-on enseignant?,} Strasbourg, Presses universitaires de Strasbourg, 1997, 236 p., bibliogr.

La création des IUFM a conduit à l'unification du recrutement et de la formation des enseignants du primaire et du secondaire. Après un rapide historique sur le développement de ces deux ordres d'enseignement, les auteurs analysent, dans une perspective sociologique, les conséquences de cette unification et le rapport que les étudiants entretiennent avec leur formation professionnelle.

CHARTIER Anne-Marie, "L'expertise enseignante entre savoirs pratiques et savoirs théoriques", Recherche et formation, $n^{\circ}$ 27, novembre 1998, p. 67-82.

Pour penser la formation professionnelle des enseignants aujourd'hui, deux modèles se trouvent en concurrence : pour le premier, une bonne diffusion des savoirs de référence est nécessaire pour orienter les choix didactiques et pédagogiques; pour le second, c'est dans l'action et par l'action que s'effectuent les progrès, qui peuvent être améliorés par une réflexion après coup. L'auteur nous livre ici ses analyses dialectiques sur la théorie et la pratique à l'aide d'une étude de cas : la pratique professionnelle d'une institutrice sur sa pédagogie de l'écriture en grande section.

GONNIN-BOLO Annette coord., BAILLAT G. coord., AUDIGIER François, LEBEAUME Joël, GREHAIGNE Jean-François, AMADE-ESCOT Chantal, BEGYN Fancis, CIFALI Mireille, PIOT Thierry, "L'identité enseignante : entre formation et activité professionnelle", Recherche et formation, $n^{\circ}$ 25, mars 1997, $121 p$.

Ce numéro est centré sur l'analyse de l'activité enseignante dans sa multiplicité, sa complexité mais aussi dans la permanence des questions qu'elle pose. Les auteurs tentent de comprendre quels peuvent être les points d'ancrage de l'identité enseignante et comment elle se construit à la fois dans les processus formels, comme les processus de formation, et dans les processus informels qui relèvent de l'activité quotidienne, des expériences, de la capacité à les analyser, à les distancier et à les réfléchir.

\section{LANG Vincent, La professionnalisation des enseignants : sens et enjeux d'une} politique, Paris, PUF (Éducation et formation. Formation permanente-education des adultes), 1999, 260 p., bibliogr.

S'interrogeant sur la nouvelle formation initiale des enseignants dans les IUFM, l'auteur engage une réflexion sur la professionnalisation des enseignants dans une perspective à la fois historique, politique, sociologique et technique; il démontre ainsi que l'on est bien en présence d'un nouveau métier, avec amélioration et valorisation du statut social, car la nature 
de la compétence professionnelle des enseignants a changé et doit maintenant se construire autour du développement de savoirs professionnels spécifiques, de la maitrise de l'expérience pratique, de l'autonomie et de la responsabilité éthique individuelle mais aussi collective.

LEGRAND Denise, "Instituteur professeur des écoles : une identité composite», Recherche et formation, $n^{\circ} 37, p$. 123-140, bibliogr.

Par delà le changement de nom, de l'instituteur au professeur des écoles, c'est à une transformation de l'image sociale et du statut qu'a conduit la création des IUFM et la mise en place d'une nouvelle formation des enseignants du premier degré. L'auteur analyse cette nouvelle identité professionnelle qui est loin d'être homogène car la nouvelle réforme, à l'origine d'une revalorisation certaine de la profession, a contribué aussi à l'éclatement du noyau identitaire.

«Les jeunes profs ou l'entrée dans la profession», Vie pédagogique, $n^{\circ} 111$, mai 1999, p. 9-40.

Dossier consacré à l'insertion professionnelle des jeunes professeurs au Québec qui connaît actuellement un fort taux de renouvellement du personnel enseignant. La réussite de l'intégration dans la carrière tient au soutien apporté dans l'exercice du métier et à la qualité de l'accueil réservé.

LESSARD Claude, CATTONAR Branka, MAROY Christian, DEMAILLY Lise, DEMBINSKI Olivier, KERROUBI Martine, VAN ZANTEN Agnès, ROBITAILLE Martin, "Nouvelles régulations et professions de l'éducation : dossier», Éducation et sociétés, $n^{\circ}$ 6, février 2000, p. 5-119.

116 Ce dossier veut analyser les effets des évolutions des politiques de l'éducation sur les organisations et sur les acteurs; il essaie aussi de mettre en relation le discours sur le "praticien réflexif» avec les changements impulsés par les politiques éducatives actuelles. L'un des articles étudie les discours actuellement tenus en Belgique francophone sur la nécessaire redéfinition du métier d'enseignant (du secondaire) à partir du modèle du praticien réflexif. Un autre met en relation l'École et l'Hôpital dans leur fonction managériale; un troisième traite de la coordination du travail dans les établissements difficiles. Le dernier article retrace l'évolution des Collèges d'enseignement général et professionnel créés au Québec dans les années 1960, à travers une typologie d'identités professionnelles.

\section{PAQUAY Léopold, ALTET Marguerite, CHARLIER Evelyne, PERRENOUD Philippe,} Former des enseignants professionnels : quelles stratégies? quelles compétences?, Bruxelles, De Boeck Université (Perspectives en éducation et formation), 2001, 268 p., bibliogr.

Cet ouvrage entend aider les formateurs d'enseignants et contribuer à la recherche sur leur propre professionnalité et celle des enseignants. La réflexion s'articule autour de trois questions cruciales : de quelle nature sont les compétences de l'enseignant-expert? Comment ces compétences professionnelles se construisent-elles? Comment organiser l'apprentissage de ces compétences professionnelles? Des pistes stratégiques et des esquisses de dispositifs, un travail sur l'analyse des pratiques et la prise de conscience sont également proposés dans cette étude qui concerne aussi bien la formation initiale que la formation continue.

PAQUAY Léopold dir., SIROTA Régine dir., «Le praticien réflexif. La diffusion d'un modèle de formation", Recherche et formation, $n^{\circ} 36$, avril 2001, p. 5-189.

Les articles de ce dossier proposent une réflexion autour de la pratique professionnelle de l'enseignant et, partant de là, de sa formation. Des travaux antérieurs ont montré que «le professionnel n'est pas un applicateur de principes théoriques ni de schémas a priori, ni de 
régles méthodologiques» et qu'il «construit son savoir professionnel par l'action et la réflexion dans et sur l'action». Ce numéro qui regroupe des chercheurs de six pays s'interroge sur la diffusion de ce modèle dans la formation des enseignants.

PERRENOUD Philippe, Développer la pratique réflexive dans le métier d'enseignant: professionnalisation et raison pédagogique, Paris, ESF (Pédagogies recherche), 2001, $219 p$.

Cet ouvrage examine comment la pratique réflexive peut influer sur la formation des enseignants et la professionnalisation de leur métier; il relie les concepts de base du paradigme réflexif, d'une part à des réflexions sur la formation en alternance, la démarche clinique, l'analyse des pratiques, d'autre part aux théories de l'action.

Université de Nantes. Centre de recherches en éducation, Professionnaliser le métier d'enseignant: savoirs professionnels et formation, Nantes, CRDP (Cahiers du CREN), 1997, 140 p.

Les articles publiés ici en présentant des recherches qui s'efforcent de rendre intelligibles certaines facettes du processus de professionnalisation, apportent des repères sur la nature des savoirs professionnels et des formations qui permettent de «développer une pratique réfléchie et autonome des enseignants et de réaliser une démocratisation véritable de l'enseignement».

\title{
L'enseignant dans sa classe : les pratiques professionnelles
}

\author{
AUDUC Jean-Louis, Agir en équipe, en collège et en lycée, Créteil, CRDP (Les \\ Guides de l'école), 1998, 150 p. \\ L'ouvrage prend pour base l'établissement d'enseignement et l'enseignant. Ce dernier est \\ considéré comme un expert dans sa discipline, mais apparaît souvent isolé. Son insertion dans \\ une équipe pédagogique, où règne la collaboration entre collègues, lui permet de faire face aux \\ problèmes rencontrés. L'auteur souligne l'importance du travail en commun.
}

\section{BARRERE Anne, Les enseignants au travail. Routines incertaines, Paris,} L'Harmattan, (Savoir et formation), 2002, 304 p.

C'est la réalité professionnelle des enseignants du secondaire qui est ici analysée. Le regard de l'auteur, sociologue, cerne les différents aspects du métier et y voit un double processus mêlant nouvelles réalités et perspectives normatives. Resitués dans une perspective historique, le statut et les diversités professionnelles sont tout d'abord évoqués, puis la préparation des cours dans le cadre des programmes où, part ailleurs, la part d'initiative et de liberté des professeurs reste entière; quelques pages sont consacrées à la culture de la discipline versus culture de la pédagogie. L'ouvrage aborde ensuite le travail d'enseignant au quotidien, dans la classe (avec le problème particulier de l'évaluation des élèves et des copies), puis dans l'établissement. La conclusion met l'accent sur l'émergence de nouvelles tâches et sur la nature du travail de l'enseignant «indissociablement routinier et incertain ».

BOUCHARD Pascal dir., VAILLE Anne-Marie, Innovation École! : de la maternelle au lycée, Paris, Autrement (Frontières), 2001, 389 p.

L'ouvrage débute sur un certain nombre d'entretiens que les deux auteurs, l'une dans le système, l'autre placé à l'extérieur, ont conduit auprès de personnalités du monde éducatif sur le thème de l'innovation; à la fin de ces interviews, ils laissent à deux spécialistes de l'innovation le soin d'en tirer les conclusions. Puis, vient le récit des pratiques innovantes sur le terrain, pratiques qui se situent dans tous les cycles du système éducatif en diversifiant au 
maximum les sujets et les régions. Quelques généralités s'en dégagent : on innove plus facilement dans le premier degré que dans les collèges ou les lycées; les ZEP et les lycées professionnels sont de bons terrains d'expérience car c'est une question de survie pour eux; et le fil conducteur des thèmes proposés peut se résumer en deux mots : "ouverture aux autres» et "autonomie». Le livre se termine sur la présentation des principaux mouvements pédagogiques.

BRU Marc coord., MAURICE Jean-Jacques coord., "Les pratiques enseignantes : contributions plurielles", Les dossiers des sciences de l'éducation, $n^{\circ} 5$, septembre 2001, 138 p.

La recherche de «bonnes» pratiques enseignantes, à travers l'étude de corpus de données, et la mesure de leurs effets est une des voies explorées en sciences de l'éducation. Ce dossier propose des contributions, selon différentes perspectives, de ces pratiques dans leur mode de construction et de réalisation. Après un débat entre chercheurs travaillant sur ce domaine, un premier article consigne réflexions sur l'effet-maître, les modalités de sa mesure et les conceptions sous-jacentes. D’autres contributions françaises et étrangères (Belgique, Suisse, Québec) analysent différentes pratiques d'enseignants dans les disciplines des mathématiques et des sciences. Les représentations sous-tendues dans ces pratiques peuvent se traduire dans la différence de traitement selon les sexes comme cela est analysé dans un des articles. Le dossier se termine par la description et l'interprétation de l'expérience d'une enseignante débutante.

CASTANY Jacqueline coord., MEARD Jacques coord., "L'autonomie de l'enseignant", Cahiers pédagogiques, $n^{\circ} 384$, mai 2000, p. 12-44, bibliogr.

La notion d'autonomie de l'élève, présente dans tous les projets d'établissement, n'est jamais accompagnée d'une réflexion sur l'autonomie de l'enseignant. Le statut particulier de l'enseignant, fonctionnaire dans une institution fortement hiérarchisée, lui permet-il de faire preuve d'autonomie et de favoriser celle de l'élève? Comment s'exerce cette autonomie, quelles formes prend-elle, quelles en sont les particularités et les limites?

\section{DEAN John, Improving Children's Learning: Effective Teaching in the Primary} School, Londres, Routledge, 2000, 190 p.

Cet ouvrage se veut une aide pour les enseignants de classe primaire afin qu'ils améliorent leurs méthodes pédagogiques et leurs savoir-faire; il fournit conseils et appui pour la conduite de la classe, pour soutenir les enfants qui ont des besoins particuliers, pour travailler en groupe et avec les parents et met à la disposition de l'enseignant les dernières recherches dans ces domaines. Il l'encourage également à devenir enseignant-chercheur sur ses propres pratiques.

GAUTHIER Clermont éd., Pour une théorie de la pédagogie : recherches contemporaines sur le savoir des enseignants, Bruxelles, De Boeck Université (Perspectives en éducation), 1997, 352 p., index, bibliogr.

S'interrogeant sur la nature d'une base de connaissances pour enseigner, une équipe de chercheurs a analysé les recherches empiriques menées dans les classes au Québec. Les résultats ont été regroupés en deux catégories : la gestion de la classe et la gestion de la matière. L'utilisation des résultats de la recherche leur permet de formuler les "prolégomènes d'une théorie empirique de la pédagogie».

GUILLAUME François-Régis, «Enseigner en ZEP : est-ce encore le même métier?», Éducation et formations, $n^{\circ}$ 61, décembre 2001, p. 75-82.

En ce qui concerne l'éducation prioritaire, les représentations des enseignants sont différentes selon que ceux-ci se situent hors ZEP ou en ZEP, en école ou en collège. Les enseignants des 
écoles s'opposent aux enseignants des collèges par une image très positive de leurs élèves et de leur niveau scolaire, d'où découle un sentiment de réussite professionnelle; mais pour tous les enseignants, l'évolution du métier est une necessité dans tous les établissements, y compris les établissements favorisés où les élèves difficiles sont minoritaires, et de ce point de vue, les ZEP sont considérées comme un vivier d'innovations et d'expérimentations.

\section{JAQUES David, Learning in groups: a handbook for improving group work, Londres, Kogan Page, 2000, 310 p.}

Cet ouvrage se veut un outil pratique, avec beaucoup d'études de cas et d'exercices, pour qu'enseignés et enseignants s'entraînent à travailler en groupe.

\section{JORRO Anne, L'enseignant et l'évaluation : des gestes évaluatifs en question, Bruxelles, De Boeck Université (Pratiques pédagogiques), 2000, 184 p., bibliogr.}

À partir des gestes de l'enseignant, cet ouvrage envisage l'évaluation comme une aide aux apprentissages des élèves et présente les diverses pratiques évaluatives en cours à l'école. La première partie aborde le rapport que l'enseignant tisse avec l'évaluation en partant des postures d'évaluateur; la deuxième partie cherche à mettre en évidence les imaginaires qui fondent les pratiques évaluatives; la troisième présente les usages scolaires de l'évaluation, ses points positifs mais aussi les difficultés de sa mise en œuvre.

LACOUR Martine, "Enseignant : un nouveau métier? ", L'Éducation enfantine, $n^{\circ}$ 6, février 2000, p. 14-18 et p. 59-65.

Le métier d'enseignant en maternelle est devenu de plus en plus complexe puisque son objectif est d'accompagner les enfants dans leur compréhension du monde. Par ailleurs, le nouveau regard porté sur les jeunes élèves nécessite un savoir hautement professionnel. Ce dossier, enrichi de statistiques et d'interviews, fait le point sur un savoir professionnel qui se situe entre continuité et rupture.

POSTIC Marcel, La relation éducative, Paris, PUF (Éducation et formation), 2001, 336 p.

L'ouvrage présente les différents modes d'analyse de la relation éducative accompagnés de commentaires critiques. L'analyse des outils conceptuels est composée de trois parties. La première est centrée sur l'analyse sociologique de l'acte éducatif, les deux autres portent sur l'approche psychologique et psychanalytique de la relation éducative. Cette neuvième édition rend compte des apports des publications récentes et présente l'influence des conceptions nouvelles de l'action pédagogique sur les formes de relation pédagogique. On y trouve aussi des contributions de spécialistes de l'éducation.

\section{RAULIN Dominique, Nouvelles technologies@nouvelle pédagogie, Paris, CNDP} (Collection de l'ingénierie éducative), 2001, 134 p. + 1 vidéocassette VHS de 70 min.

Composé d'un livre et d'une vidéo, ce document montre comment l'ordinateur, longtemps considéré comme réservé aux élèves en difficulté, est aujourd'hui un outil au service des apprentissages de tous les élèves et comment les TICE offrent de nouvelles possibilités au système éducatif pour s'ouvrir vers l'extérieur, aux enseignants pour exercer leur métier, aux élèves pour apprendre.

\section{BERBAIN Jean-Marie, CAUJOLLE Monique, ÉTÉVÉ Christiane dirs., Repères} pour enseigner aujourd'hui, Paris, INRP (Chercheurs et enseignants), 1999, 168 p.

Ce livre propose une réflexion sur différents thèmes liés à l'exercice du métier d'enseignant: méthodes d'apprentissage, travail en équipes, orientation, formation continue... Il présente la réalité scolaire éclairée par les travaux les plus récents menés par les chercheurs. 


\section{TARDIF Maurice, LESSARD Claude, Le travail enseignant au quotidien,}

Bruxelles, De Boeck, 1999, 600 p.

En menant directement leur enquête dans les établissements scolaires, les auteurs ont voulu répondre à un cerain nombre d'interrogations à propos de l'activité enseignante; que signifie travailler comme enseignant aujourd'hui? existe-t-il des savoirs, une technologie ou des outils propres à ce métier? Cet ouvrage se situe aussi dans une perspective internationale par ses études comparatives entre divers systèmes d'enseignement.

\section{Perspectives}

BOTTIN Yves, Enseigner en école, un métier pour demain : rapport au ministre de l'éducation nationale, février 2002, 54 p. téléchargées du site: www.education. gouv.fr/ Ces derniers temps, les séminaires et les missions de réflexion sur le métier d'enseignant se font de plus en plus nombreux, qu'ils soient suscités par le ministère de l'Éducation nationale ou les partenaires sociaux comme le SNES ou la chambre de commerce et d'industrie de Paris. Ici, le rapport concerne les évolutions du métier d'enseignant du premier degré en face des mutations de la société et de ses attentes. L'auteur préconise la réorganisation du réseau des écoles et la création des établissements primaires publics locaux d'enseignement (EPPLE), à l'image des EPLE du second degré; il s'interroge sur la carrière et les parcours professionnels offerts aux professeurs des écoles, sur l'organisation et la pratique du métier, sur la polyvalence des maîtres et leur temps de travail. En conclusion, il est rappelé que, face à la demande sociétale, l'école primaire doit passer d'une obligation de moyens, en grande partie atteinte, à une obligation de résultats que l'institution se doit d'assumer.

CCIP : Chambre de Commerce et d'Industrie de Paris, Enseigner demain : des enseignants qui innovent pour un métier qui change, février 2002, 15 p. téléchargées du site: www.ccip.fr/etudes/friedland/fri0202.htm.

La CCIP, qui a de fait une mission éducative, se penche lors de ces entretiens sur les acteurs de la formation; les évolutions de la société, les attentes des entreprises leur imposent le défrichement de voies nouvelles, pour les méthodes comme pour les programmes ou les dispositifs de formation. Sur ce thème, après une introduction faite par le directeur du CERI, plusieurs invités interviennent, venus du monde enseignant comme du monde industriel.

\section{CORNU Bernard dir., Commission nationale française pour l'Unesco, Le nouveau métier d'enseignant, Paris, La Documentation française, 2000, 44 p.}

La société change, l'école change, les élèves changent; et l'enseignant est au cœur de ces changements: il les vit, les subit parfois, il les accompagne mais peut aussi les anticiper. Cet ouvrage, fruit d'un travail collectif conduit dans le cadre de la commission française pour l'UNESCO, tente d'analyser ces évolutions du métier d'enseignant en se plaçant dans une perspective internationale. La première partie de l'ouvrage est consacrée au rapport du groupe de travail qui, après un rappel historique, cherche à définir le rôle majeur de l'enseignant aujourd'hui et à formuler quelques propositions novatrices ainsi que des recommandations de portée internationale. La deuxième partie représente la synthèse de la table ronde sur le thème du «nouveau métier d'enseignant».

CORNU Bernard, BRIHAULT Jean, Pour une rénovation du dispositif de formation des enseignants : rapport à monsieur le ministre de l'Éducation nationale, janvier 2002, 25 p. téléchargées du site: www.ferc.free.fr/Composantes/ Unsen/Iufm/Actualités/renovation_des_iufm_2001.htm 
Ce rapport a pour objet, dix ans après la création des IUFM de proposer des dispositions pour rénover et améliorer le dispositif de formation des enseignants; il entend répondre à deux questions majeures: comment mieux recruter les enseignants dont le système éducatif a besoin? Comment assurer aux futurs enseignants une meilleure formation professionnelle?

"La nouvelle vague : coup de jeune chez les profs», Monde de l'éducation (Le), $n^{\circ} 282$, juin 2000, p. 21-40.

D'ici dix ans, $40 \%$ des enseignants aujourd'hui en poste seront remplacés par leurs jeunes confrères. L'école doit tout faire pour que le rajeunissement des effectifs soit l'opportunité d'une transformation réussie.

MOTIVANS Albert, BROSSARD Mathieu, LYND Douglas, SCHLEICHER Andreas, BRUNEFORTH Michael, SINISCALCO Maria Teresa, TREMBLAY Karine, UNESCO, Teachers for tomorrow's schools: analysis of the World Education Indicators, Paris, UNESCO; Paris, OCDE; Washington, World Bank, 2001, 226 p.

Dans l'effort d'éducation qu'a entrepris un grand nombre de pays depuis la dernière décennie, le rôle des enseignants n'a fait que s'accroître; les gouvernements en ont-ils pris conscience? Cet ouvrage tente de répondre à cette interrogation et accorde une attention particulière aux enseignants, à leurs demandes, leurs motivations, leur salaire et leur carrière à travers une étude portant sur seize pays participant au programme World Education Indicators (WEI). Le premier chapitre brosse l'environnement macro-économique de ces pays; le second examine ce réel besoin d'enseignants et l'implication financière que cela suppose. Le troisième chapitre dresse, pays par pays, un tableau statistique des facteurs déterminants dans cette demande d'enseignants qualifiés.

OBIN Jean-Pierre, Enseigner, un métier pour demain : rapport au ministre de

l'Education nationale, mars 2002, 87 p. téléchargées du site: www.education. gouv.fr/rapport/obin.pdf

La mission qui a donné lieu à ce rapport sur les enseignants du second degré s'est organisée autour de six axes complémentaires: une étude historique, des synthèses des travaux de recherche sur les jeunes et les professeurs du secondaire, une étude internationale (comment nos partenaires de l'OCDE affrontent-ils ce problème), des enquêtes et des entretiens auprès d'étudiants et d'enseignants, une étude organisée par le groupe d'experts «Nouvelles pratiques d'enseignement et d'éducation ", des auditions de personnalités et de responsables d'organisation. Le rapport, lui, est structuré en deux grandes parties; la première partie fait l'historique de la profession d'hier à aujourd'hui; la deuxième partie est plus tournée vers l'avenir en abordant la question du recrutement, de l'attractivité du métier et de son adéquation avec les aspirations de la société, celle aussi de la gestion, de l'évaluation des enseignants et de l'aide que l'institution peut leur apporter. La conclusion fait ressortir la nécessité de piloter autrement.

TAYLOR David, Conseil de l'Europe. Conseil de la coopération culturelle. Un enseignement secondaire pour l'Europe, Le développement des ressources humaines pour l'enseignement secondaire en Europe : les personnels enseignants et non enseignants - aujourd'hui et demain, Strasbourg, Conseil de l'Europe, 1996, 33 p.

Le thème du séminaire a conduit les participants à aborder des aspects fondamentaux de la vie professionnelle des enseignants, et à discuter de la nature de la collectivité scolaire et des politiques nationales et supranationales d'éducation. Les objectifs suivants ont été définis: étudier les liens entre les profils de formation actuels des personnels enseignants et non enseignants et leurs fonctions et responsabiblités effectives; faire ressortir les nouveaux besoins des écoles, des élèves et de la société en général; mettre en évidence les tendances et les innovations dans le développement des ressources humaines dans les écoles. 
TEDESCO Juan Carlos éd., "Le rôle des enseignants dans un monde en changement: dossier", Perspectives (Unesco), vol. XXVI, n 3, septembre 1996, p. 471-612, bibliogr.

Les différents articles rassemblés dans ce dossier visent à identifier les principaux facteurs que doivent prendre en compte les stratégies de transformation du rôle de l'enseignant. La massification de la profession s'est parfois accompagnée d'une dégradation du recrutement. Le métier d'enseignant évolue et ses nouvelles exigences nécessitent une formation adaptée. Ces changements doivent eux-mêmes s'inscrire dans la gestion globale du système éducatif. 